\title{
Research on Evaluation Index Weight Under the Condition of Random WANG Xuefeng
}

\author{
Chongqing College of Electronic Engineering, Chongqing, 401331, China \\ E-mail: moe361@163.com
}

Key words: index weight; random variables; uniform distribution

\begin{abstract}
In the process of economic evaluation, the determination of the weight of each index in the index system is the focus and difficulty of the evaluation work. There are many studies on the determination of weight in various literature, but each has its advantages, disadvantages and limitations in specific application. In this paper, by calculating the expectation and variance, we discuss the normalization and non-normalization of weights, and illustrate the arithmetic by numerical examples, which provides a method and idea for simplifying the evaluation process.
\end{abstract}

\section{Introduction}

In scientific research, we often establish evaluation index system to evaluate the effect of events or a project. Typically research focused on identifying the weights of each evaluation index, and for quantitative evaluation focuses on determining the weights through the calculated results of the evaluation. At present there are many ways in determining index weight, but there are a lot of problems need to be discussed when the index weight is random variable. In this paper, by analyzing a variety of methods, using the concept of expectation and variance of probability directly to calculate the index weight for the evaluation results under random variable situation, thus to put forward the new thinking of the evaluation work instead.

\section{Research review}

In determining the index weight, the commonly used methods are the subjective weighting method, the objective weighting method, the combination weighting method. In recent years, many scholars have been seeking for weight assignment Method, such as $\pi$ PV Method set up by Wang Lingfeng ${ }^{[1]}$, a combination of Paired Comparison (Paired Comparison Method) and the Maximum Variance Method (Maximum Variance Method), the method is used to solve employee performance evaluation system; Su Weihua ${ }^{[2]}$ set up a new method which combined with AHP method and Delphi method used in the statistical constitutive power expert opinion divergence degree index weight; Liu Hong ${ }^{[3]}$ comprehensively considerate the AHP method, principal component analysis, grey correlation method, three methods of synthesis is applied to the determination of index weight. Ceng Wenyi ${ }^{[4]}$ introduced a method for interval Numbers - line method, this method is inviting 3 to 5 experienced experts, each expert gave satisfactory interval, finally, these intervals be gone and set the number as the last interval. Gao Fengji put forward the concept of feasible weighting interval ${ }^{[5]}$, the expert analysis of various index feasible weighting interval as limiting conditions, uses linear programming to find the optimal solution.

Summarize various weight calculation method and evaluation system of the practical situation, when evaluating an index, the weight is not an accurate numerical or completely rely on to calculate, also need to rely on statistics or experience to comprehensive treatment. So in this case we may consider the weight as a random variable, think it on values in a certain interval in accordance with a certain distribution, then using the related mathematics knowledge to the evaluation results of needed to calculate directly, the expectation and variance of the evaluation results. 


\section{The reason the index random weight has arisen}

For the randomness of index weight, it is likely to appear in the following four cases:

(1) When determining certain index weight, when comparing a number of indicators to quantitatively and normalized calculation, most experts agree that it is difficult to give quantitative score and calculate the weight. We think we can according to the statistical data or experience in distribution function to represent the weight of the index;

(2) When determining certain index weight, mark to an index by a number of experts is quick difference, cannot be used directly for the determination of weight, we can consider to give a distribution interval to define the weight of the index;

(3) When using the objective weighting method, for different sample in the index system, using the same method to determine the weight is quite differences, we can combine the sample here to consider the weight distribution of a given interval to calculate the index weight;

(4) As the evaluation index system is a comprehensive and general index system, and in use process is targeted at specific projects or factors, etc., it should be adjusted in a kind of evaluation index, increase or reduce the number of single index, it should be considered at this time in the class of other individual indicators weight to adjust, interval would be more appropriate for a reference.

\section{Discussion on the weight for the random variable case}

For a weighted comprehensive system $T=\sum_{i=1}^{n} w_{i} P_{i}$, among them $\sum_{i=1}^{n} w_{i}=1, w_{i}>0,(i=1,2, \ldots, n)$, If the part weight is random, that is, some $w_{i}$ is a random variable (If it is a random variable, the following with capital letters ), and obey a certain distribution, such as the [0,1] uniform distribution and Beta distribution range, etc, and in the following discussion, considering the characteristics of the index weight, usually we take the uniform distribution method to discuss. Now consider the index weight for the random cases, how to calculate the index distribution, characteristics (such as the mean, variance, etc.).

Situation (1): when $n=2$

Assuming that $W_{i} \sim F_{i}(x), i=1,2, \quad f_{i}(x)(i=1,2)$ is density function for them, that is when $x<0$ or $x>d_{i}, f_{i}(x)=0$, among them $0 \leq d_{i} \leq 1$.

In order to ensure the weight normalized, we may assume that two random weight has dependency, that is $W_{1}=1-W_{2}$ or normalization processing for the realization of the weighting value, as shown in the following formula, and the new weight is:

$$
W_{1}^{(1)}=\frac{W_{1}}{W_{1}+W_{2}}, W_{2}^{(1)}=\frac{W_{2}}{W_{1}+W_{2}}
$$

Among them $W_{i}^{(1)},(i=1,2)$ is the normalized weight of index 1 and 2 , so in order to facilitate discussion, might as well always hypothesis $W_{1}=1-W_{2}$ was established.

Because $T=W_{1} P_{1}+W_{2} P_{2}=W_{1} P_{1}+\left(1-W_{1}\right) P_{2}=W_{1}\left(P_{1}-P_{2}\right)+P_{2}$

So $T$ distribution density function is:

$$
f_{T}(t)=\left\{\begin{array}{lc}
\frac{1}{\left|P_{1}-P_{2}\right|} f_{1}\left(\frac{t-P_{2}}{P_{1}-P_{2}}\right), & \text { when }\left(t_{1}<t<t_{2}\right), \\
0, & \text { else. }
\end{array}\right.
$$

Among them $t_{1}=\min \left\{P_{2}, d_{1}\left(P_{1}-P_{2}\right)+P_{2}\right\}, t_{2}=\max \left\{P_{2}, d_{1}\left(P_{1}-P_{2}\right)+P_{2}\right\}$.

One hand, $E[T]=E\left[W_{1} P_{1}+W_{2} P_{2}\right]=E\left[W_{1}\right] P_{1}+E\left[W_{2}\right] P_{2}$,

And other hand, $E[T]=E\left[W_{1}\right]\left(P_{1}-P_{2}\right)+P_{2}$.

So that if $P_{2} \neq 0$, obtain $E\left[W_{1}\right]=0.5$, and variance can be obtained 
$\operatorname{Var}[T]=\operatorname{Var}\left[W_{1}\right]\left(P_{1}-P_{2}\right)^{2}$.

For example, take the uniform distribution,

It is $(i=1,2), f_{i}(x)=\left\{\begin{array}{l}\frac{1}{d_{i}}, \text { when } 0<x<d_{i}, \\ 0, \quad \text { else. }\end{array}\right.$.

Because $E\left[W_{1}\right]=0.5$, so $d_{i}=1$, it is

$$
f_{T}(t)=\left\{\begin{array}{lc}
\frac{1}{\left|P_{1}-P_{2}\right|}, & \text { when } \min \left\{P_{2}, P_{1}\right\}<t<\max \left\{P_{2}, P_{1}\right\}, \\
0, & \text { else }
\end{array}\right.
$$

That is when $\min \left\{P_{2}, P_{1}\right\}<t<\max \left\{P_{2}, P_{1}\right\} \quad T$ is uniform distribution, the averages of $T$ is $E[T]=\frac{P_{1}+P_{2}}{2}$, the variance of $T$ is $\operatorname{Var}[T]=\frac{\left(P_{1}-P_{2}\right)^{2}}{12}$.

Situation (2): when $n \geq 3$,

Assuming that $W_{i} \sim F_{i}(x), i=1,2, \ldots, m, f_{i}(x)$ is density function for them, that is when $x<0$ or $x>d_{i}, f_{i}(x)=0$, among them $0 \leq d_{i} \leq 1$.

Because $W_{1}+W_{2}+\cdots+W_{m}+w_{m+1}+\cdots+w_{n}=1$ ( Here we assume that the first $m$ weight is random variables, after the $n-m$ weight is constant.)

Therefore $w_{\Sigma}=\sum_{i=m+1}^{n} w_{i}$.

So, we need to consider: $W_{1}+W_{2}+\cdots+W_{m}=1-w_{\Sigma}$, and $0<w_{\Sigma}<1$.

Assuming each $W_{i},(i=1,2, \ldots, m)$ obey a uniform distribution on $\left[0, d_{i}\right]$, there is constraints:

$$
\sum_{i=1}^{m} d_{i}=2\left(1-w_{\Sigma}\right)
$$

At this time,

$$
E[T]=\sum_{i=1}^{m} E\left[W_{i} P_{i}\right]+\sum_{i=m+1}^{n} w_{i} P_{i}=\sum_{i=1}^{m-1} \frac{d_{i} P_{i}}{2}+\left(1-w_{\Sigma}-\frac{\sum_{i=1}^{m-1} d_{i}}{2}\right) P_{m}+\sum_{i=m+1}^{n} w_{i} P_{i} .
$$

The variance is:

$$
\operatorname{Var}[T]=\operatorname{Var}\left[\sum_{i=1}^{m-1} W_{i}\left(P_{i}-P_{m}\right)\right]=\sum_{i=1}^{m-1} \frac{d_{i}^{2}\left(P_{i}-P_{m}\right)^{2}}{12} .
$$

For $W_{1}+W_{2}+\cdots+W_{m} \neq 1-w_{\Sigma}$, the implementation of the weight normalized values, such as shown in the following equation, the new weight is

$$
\begin{array}{cc}
W_{i}^{(1)}=\frac{W_{i}}{W_{1}+W_{2}+\cdots+W_{m}+1-w_{\Sigma}}, & i=1,2, \ldots, m . \\
w_{j}^{(1)}=\frac{w_{j}}{W_{1}+W_{2}+\cdots+W_{m}+1-w_{\Sigma}}, & j=m+1, m+2, \ldots, n .
\end{array}
$$

If $W_{i},(i=1,2, \ldots, m)$ is uniform distribution random variable on independent of ${ }^{\left[0, d_{i}\right]}$, we can give the following conclusion: 


$$
T=\frac{\sum_{i=1}^{m} W_{i} P_{i}+\sum_{j=m+1}^{n} w_{j} P_{j}}{\sum_{i=1}^{m} W_{i}+\sum_{j=m+1}^{n} w_{j}}
$$

So you can discuss the distribution and characteristics values of $T$, but usually more complicated analytical solution is given, you can use Monte-Carlo method for numerical calculation.

\section{Conclusions}

Through the above discussion, we got under the condition of the index weight for the random variable how to calculate the upper evaluation results, the method of this and previous compared all kinds of random weight method, simplifies the calculation process, also can be properly adjusted according to actual condition, more make evaluations on the whole grasp and understanding of the evaluation results, fully combines the actual conditions of the index application purpose, can more fully reflect the value of the evaluation index system.

\section{Corresponding author}

Wang Xuefeng, male, Hebei Yangyuan, professor, research direction for Industrial Engineering, logistics management. Tel.: 0086-13650569315. E-mail address: moe361@163.com, Chongqing College of Electronic Engineering, Chongqing, 401331

\section{References:}

[1] Wang Lingfeng. New method of principle and examples of Employee performance[J]. Journal of China human resources development, 2002, 4: 33-35.

[2] $\mathrm{Su}$ Weihua. Design of expert opinion divergence indicator of Delphi-AHP statistical constitutive weight [J]. Statistical Research, 2004, 1: 31-34.

[3] Liu Hong. The study of the method to determine index weight of the comprehensive evaluation [J]. Journal of hebei university of technology, 1996, 4 (25): 75-80.

[4] Ceng Wenyi, Luo Chengzhong. Comprehensive decision-making model of interval Numbers [J]. Journal of systems engineering theory and practice, 1997, 11:48-50.

[5] Gao Fengji, Huang Yongfang, Ren Xiaoyan.Method of the Ideal Point Close to the Degree of Interval of Multiple Attribute Decision Making[J]. Journal of quantitative technical economics, 2001.2:58-61. 